\title{
Novel transparent ceramics for solid-state lasers
}

\author{
Hao Yang ${ }^{1}$, Jian Zhang ${ }^{1}$, Dewei $\mathrm{Luo}^{2}$, Hui Lin ${ }^{2}$, Deyuan Shen ${ }^{1}$, and Dingyuan Tang ${ }^{1,2}$ \\ ${ }^{1}$ School of Physics and Electronic Engineering, Jiangsu Normal University, Xuzhou 221116, China \\ ${ }^{2}$ School of Electrical and Electronic Engineering, Nanyang Technological University, Singapore 639798, Singapore \\ (Received 13 October 2012; revised 20 November 2012; accepted 2 August 2013)
}

\begin{abstract}
Recent progress on rare-earth doped polycrystalline YAG transparent ceramics has made them an alternative novel solid-state laser gain material. In this paper, we present results of our research on polycrystalline RE:YAG transparent ceramics. High optical quality YAG ceramics doped with various rare-earth (RE) ions such as $\mathrm{Nd}^{3+}, \mathrm{Yb}^{3+}, \mathrm{Er}^{3+}$, $\mathrm{Tm}^{3+}$, and $\mathrm{Ho}^{3+}$ have been successfully fabricated using the solid-state reactive sintering method. Highly efficient laser oscillations of the fabricated ceramics are demonstrated.
\end{abstract}

Keywords: RE:YAG; Solid State Lasers; Transparent Ceramics

\section{Introduction}

Recently, there has been a resurgence of interest in rareearth (RE) doped YAG (RE:YAG) transparent ceramics as laser gain media, because the optical quality of the ceramics has been greatly improved. It is now comparable to that of single-crystal materials ${ }^{[1,2]}$. Compared with singlecrystal-based laser materials, apart from possessing exactly the same spectroscopic properties, ceramic laser materials also have the advantages of ease to fabricate large size and complicated structures, short fabrication time, and the possibility of mass production.

Polycrystalline ceramics became available for optical applications in 1962, when Coble first prepared translucent $\mathrm{Al}_{2} \mathrm{O}_{3}$ ceramics ${ }^{[3]}$. Since then, various kinds of transparent ceramics have been developed for optical and related applications. In 1995, Ikesue et al. achieved highly efficient continuous-wave $(\mathrm{CW})$ laser emission in a Nd:YAG transparent ceramic ${ }^{[4-6]}$. They fabricated transparent polycrystalline Nd:YAG ceramics by a solid-state reactive sintering method using high-purity powders as the starting materials. A laser output of about $70 \mathrm{~mW}$ was obtained with a slope efficiency of $28 \%$. Their result clearly demonstrated the potential high functionality and flexibility of the laser ceramics. Since 1999, Yagi's group in Konoshima Chemical and Ueda's group in the University of Electrocommunications, Japan, have developed highly transparent ceramic laser materials in different ways: using nanopowder technology and using a vacuum sintering method. Scattering losses were greatly reduced in Konoshima's YAG transpar-

Correspondence to: Dingyuan Tang, 101 Shanghai Road, Tongshan District, Xuzhou 221116, Jiangsu, China. Email: edytang @ntu.edu.sg ent laser ceramics ${ }^{[7,8]}$. In 2000 and 2001, Lu et al. reported the operation of diode pump Nd:YAG ceramic lasers with ceramic samples made by Konoshima Chemical. The output power was gradually increased from $31 \mathrm{~W}$ to $110 \mathrm{~W}^{[8-11]}$. Extensive studies have shown that the thermal and mechanical properties of the ceramics are better than those of YAG single crystals ${ }^{[12,13]}$. In 2006, the Lawrence Livermore National Laboratory (LLNL) succeeded in achieving a world record high power of $67 \mathrm{~kW}$ emission from its solid-state heat capacity laser system using a large-scale transparent $\mathrm{Nd}$ :YAG ceramic with samarium edge cladding supplied by Konoshima Chemical ${ }^{[14]}$. Very recently, the emission of a $\mathrm{Nd}$ :YAG ceramic laser system with output power beyond $100 \mathrm{~kW}$ was further demonstrated ${ }^{[15-17]}$.

In the past years, most of the research work on transparent laser ceramics has focused on Nd:YAG ceramics. Recently, some research groups have also paid attention to developing other rare-earth doped YAG ceramics, such as $\mathrm{Yb}: \mathrm{YAG}$, Er:YAG, Ho:YAG, the sesquioxide ceramics $\left(\mathrm{Y}_{2} \mathrm{O}_{3}, \mathrm{Lu}_{2} \mathrm{O}_{3}, \mathrm{Sc}_{2} \mathrm{O}_{3}\right)$, and composite ceramics. Dong et al. reported continuous-wave and passively Q-switched microchip lasers made of Yb:YAG ceramics ${ }^{[18]}$. Heavily doped Yb:YAG ceramics are more suitable for thin disk lasers than Yb:YAG single crystal. Sesquioxides possess higher thermal conductivities than YAG. For high-power lasers, thermal conductivity is an important parameter of the laser gain media. Kim et al. reported the fabrication of $\mathrm{Yb}: \mathrm{Lu}_{2} \mathrm{O}_{3}$ transparent ceramic by a hot isostatic pressing (HIP) approach ${ }^{[19]}$. They successfully demonstrated efficient lasing with fabricated $\mathrm{Yb}: \mathrm{Lu}_{2} \mathrm{O}_{3}$ ceramic. An output power of more than $16 \mathrm{~W}$ at $1080 \mathrm{~nm}$ was obtained with a slope efficiency of $74 \%$. Ikesue et al. demonstrated laser- 


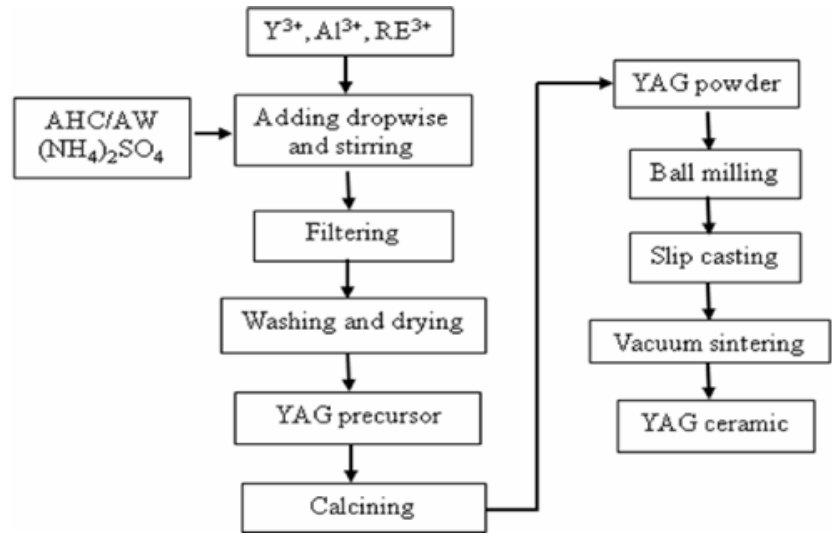

Figure 1. Fabrication process of YAG ceramic by co-precipitation and vacuum sintering method.

quality composite transparent ceramics by using a layer by layer approach ${ }^{[2]}$. Messing et al. developed a colloidal tape casting process to make composite laser ceramics. A ceramic rod with segments of undoped YAG, $0.25 \%$ Er:YAG, and $0.5 \%$ Er:YAG was fabricated. With such a composite ceramic rod of length $62 \mathrm{~mm}$, lasing with a slope efficiency of $27 \%$ was achieved, compared with the $39 \%$ slope efficiency achieved on a commercial $45 \mathrm{~mm}$ long $0.5 \%$ Er:YAG single crystal rod ${ }^{[20]}$.

\section{Fabrication of YAG laser ceramics}

Suitable fabrication methods are required to obtain YAG transparent ceramics of $>99.99 \%$ density. So far, two different approaches have been successfully implemented to produce high-quality laser ceramics. Figure 1 shows the fabrication process for YAG transparent ceramics by the coprecipitation and vacuum sintering method ${ }^{[21]}$. Konoshima Chemical succeeded in fabricating high-quality transparent YAG-based laser ceramics using this method. The method is based on co-precipitation of a metal nitrate solution with $\mathrm{NH}_{4} \mathrm{HCO}_{3}$. Aqueous solutions of aluminum, yttrium and rare-earth nitrates are mixed together. The mixed aqueous solution is added dropwise and mixed with an aqueous solution of $\mathrm{NH}_{4} \mathrm{HCO}_{3}$. Steps of filtration and washing with water are repeated several times, and the resulting material is then dried for 2 days at $\sim 120^{\circ} \mathrm{C}$. The precursor thus obtained is calcined at $1200^{\circ} \mathrm{C}$, and then the YAG powder is obtained. This powder is milled with solvent, binder, and dispersion medium for 24 hours. The milled slurry is put in a gypsum mold and dried to obtain the desired form, or the slip casting forming method is used to obtain a high-density and uniformly green body. After removing the organic component by calcining, such material is vacuum sintered at $1700^{\circ} \mathrm{C}$; then highly transparent $\mathrm{Nd}$ :YAG ceramics can be obtained.

Ikesue reported laser-quality polycrystalline $\mathrm{Nd}$ :YAG transparent ceramics made using a reactive sintering method. Figure 2 shows the fabrication process of the method ${ }^{[4]}$.

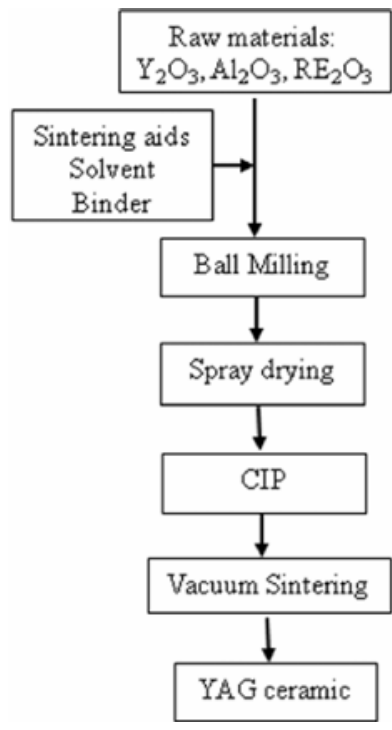

Figure 2. Fabrication process of YAG ceramic by reactive sintering method.

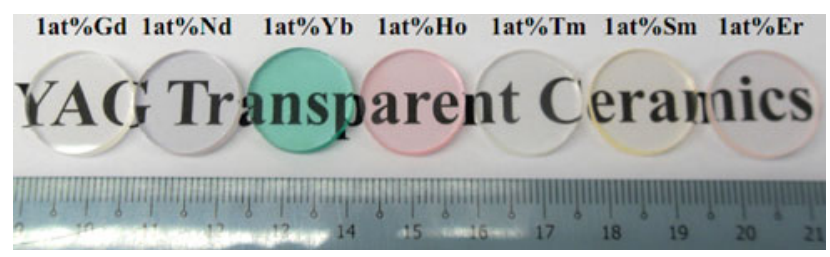

Figure 3. Photograph of different rare-earth doped YAG transparent ceramics fabricated in-house.

Commercial $\alpha-\mathrm{Al}_{2} \mathrm{O}_{3}, \mathrm{Y}_{2} \mathrm{O}_{3}$, and $\mathrm{RE}_{2} \mathrm{O}_{3}$ powders are selected as the starting materials. The powders are weighed precisely according to the chemical stoichiometric composition and mixed with $99.99 \%$ ethanol. The mixed slurry is then ball milled using a planetary milling machine for 15 hours. TEOS is introduced at $0.5 \mathrm{wt} \%$ relative to the total powder mass to introduce $\mathrm{SiO}_{2}$ as a sintering aid. After milling, the powder mixture is dried at $120^{\circ} \mathrm{C}$ for 24 hours in an oven and then sieved through a 200 -mesh screen. After removing organic components by calcining at $800^{\circ} \mathrm{C}$ for 3 hours, the powders are dry pressed in a stainless steel die at $15 \mathrm{MPa}$. The green body pellets are further cold isostatically pressed (CIP) at $200 \mathrm{MPa}$. After CIP, the relative density of the green body is $\sim 53 \%$. The green bodies are sintered in a high temperature vacuum sintering furnace. The sintered pellets are then annealed in air at $1400^{\circ} \mathrm{C}$ for 15 hours to completely remove internal stress and eliminate the oxygen vacancies. After polishing, the specimen can be used as a laser medium.

\section{Properties of the RE:YAG ceramics}

Using the reactive sintering method, we have succeeded in fabricating high-quality transparent YAG ceramics doped with different rare-earth ions such as $\mathrm{Nd}^{3+}, \mathrm{Yb}^{3+}, \mathrm{Er}^{3+}$, 

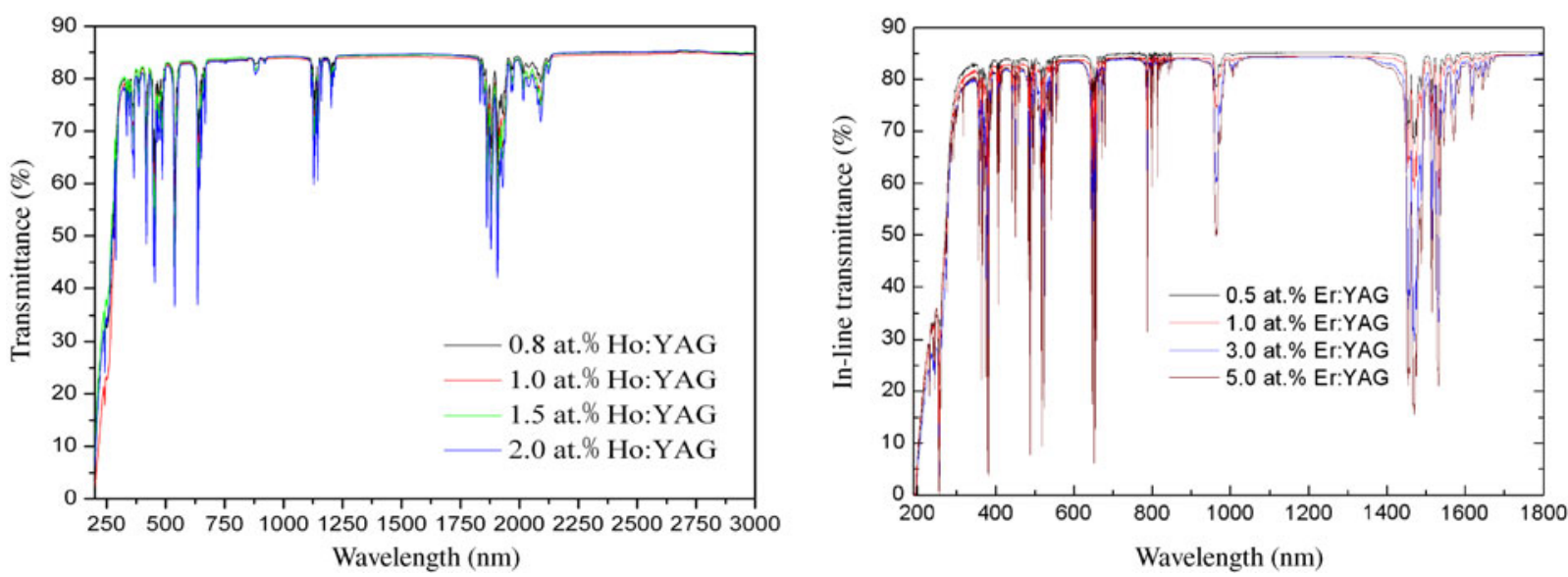

Figure 4. The in-line transmittance of Ho:YAG and Er:YAG transparent ceramics with different doping concentrations.
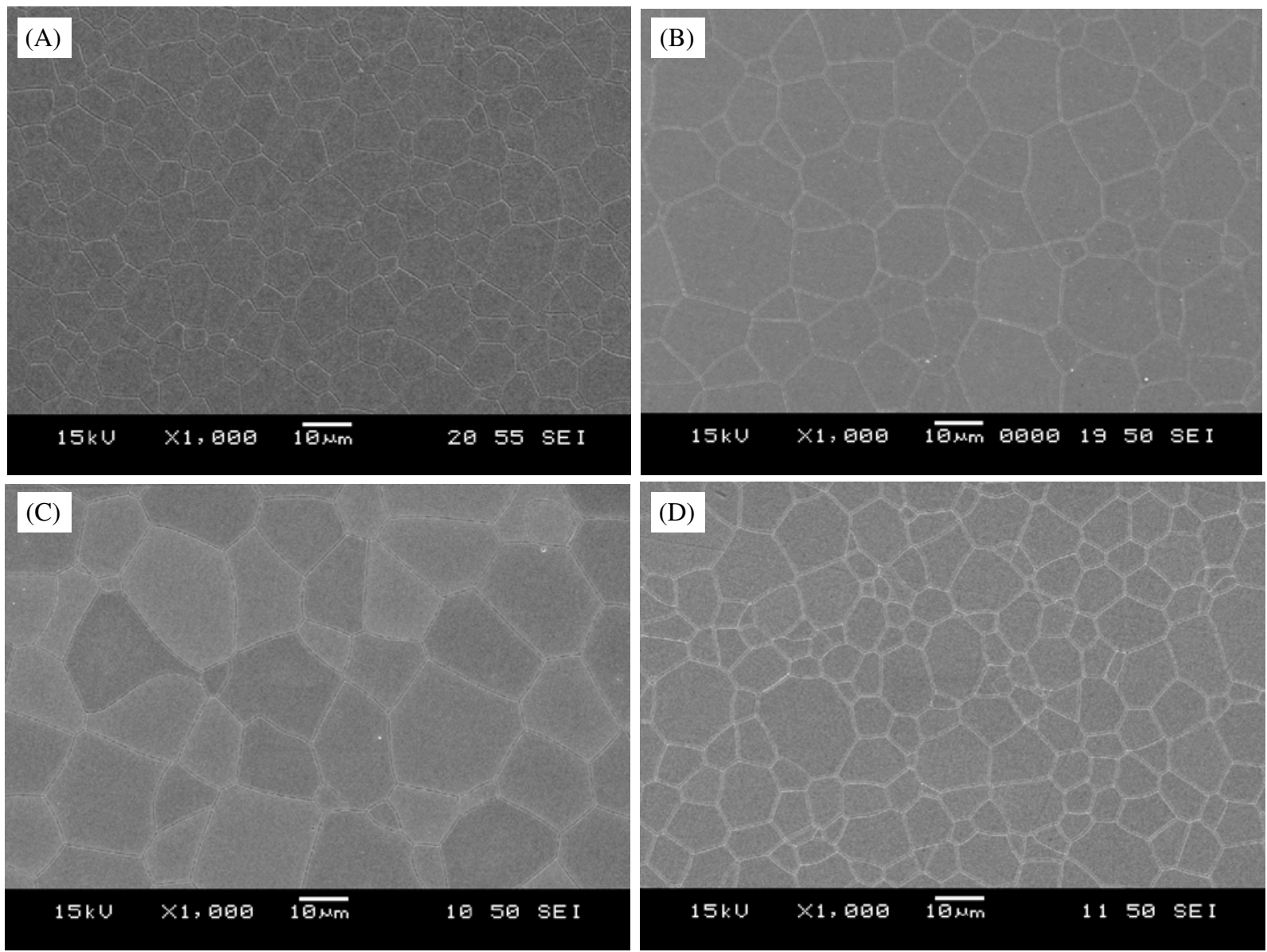

Figure 5. The microstructures of the RE:YAG ceramics fabricated in-house: (A) Er:YAG, (B) Ho:YAG, (C) Tm:YAG, and (D) Nd:YAG.

$\mathrm{Ho}^{3+}$ and $\mathrm{Tm}^{3+}{ }^{[22-28]}$. Figure 3 shows a photograph of various rare-earth-ion doped YAG ceramics fabricated inhouse. To illustrate the high optical quality of the fabricated YAG ceramics, as examples, we have shown in Figure 4 the optical transmission of $\mathrm{Er}^{3+}$ and $\mathrm{Ho}^{3+}$ doped YAG under different doping concentrations. The transmittance of all the ceramics at $1100 \mathrm{~nm}$ is above $84 \%$, which is very close to the theoretic transmittance.

Grain sizes, grain-boundary phases, and pores are usually considered as the main factors affecting the optical quality of transparent ceramics. Figure 5 shows the microstructures of YAG ceramics doped with different types of rare-earth ion after thermal etching at $1500^{\circ} \mathrm{C}$ for 1 hour. The transparent 

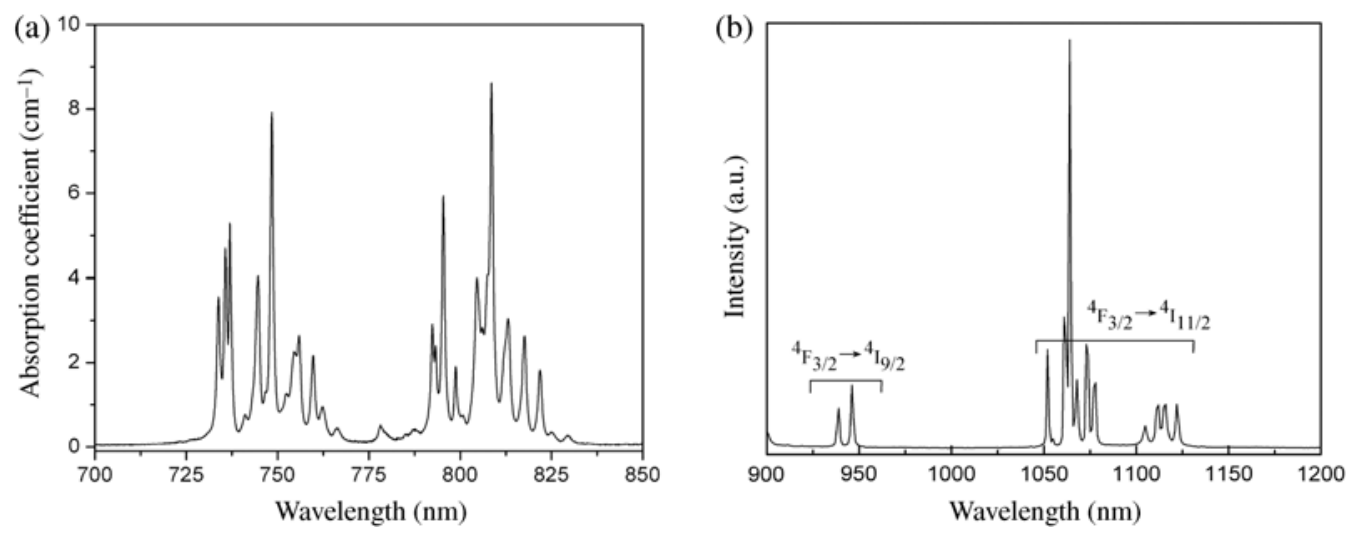

Figure 6. The absorption and emission spectra of the fabricated Nd:YAG ceramics.

ceramics are composed of grains with an average size $\sim 10 \mu \mathrm{m}$. It can be seen that the grain size changed very little with the different types of rare-earth-ion doping. No grain-boundary phases and pores can be observed in the microstructures of the RE:YAG ceramics.

\section{Laser performance of the fabricated ceramics}

There has been extensive research on the lasing performance of Nd:YAG and $\mathrm{Yb}: \mathrm{YAG}$ ceramics, not only because high optical quality Nd:YAG ceramics were successfully produced, but also because their single-crystal counterparts have been widely used in commercial solid-state laser systems. $\mathrm{Nd}^{3+}$ or $\mathrm{Yb}^{3+}$ doped lasers emit in the $1.0 \mu \mathrm{m}$ wavelength regime. High-power solid-state lasers operating in this wavelength regime have found widespread applications in various fields ranging from industrial materials processing to medical devices. Compared with $\mathrm{Nd}^{3+}$ and $\mathrm{Yb}^{3+}$ ion doped YAG ceramics, $\mathrm{Er}^{3+}, \mathrm{Tm}^{3+}$, and $\mathrm{Ho}^{3+}$ doped YAG ceramics emit in the $1.5-2.0 \mu \mathrm{m}$ wavelength regime. 1.5-2.0 $\mu \mathrm{m}$ laser emission is considered eye-safe, as the cornea absorbs wavelengths greater than $1.4 \mu \mathrm{m}$. Light with shorter wavelengths can reach the more sensitive retina. Therefore, the potential of eye damage by the scattered laser beam is much lower for lasers operating at wavelengths above $1.4 \mu \mathrm{m}$. Eye-safe solid-state lasers have applications such as in the range finders, remote sensing, and medical devices.

Using the in-house fabricated RE:YAG transparent ceramics, we designed ceramic lasers operating at different wavelengths. Our main purpose is to study the laser performance of the fabricated laser ceramics.

\section{1. $1.0 \mu \mathrm{m}$ laser oscillation}

Figure 6 shows the absorption and emission spectra of a 1.0 at.\% Nd:YAG ceramic fabricated in-house. Compared with those measured with a Nd:YAG single crystal, no measurable difference could be identified. The absorption peak of the ceramic is located at $808 \mathrm{~nm}$, and the peak absorption coefficient is measured as $8.64 \mathrm{~cm}^{-1}$, which corresponds

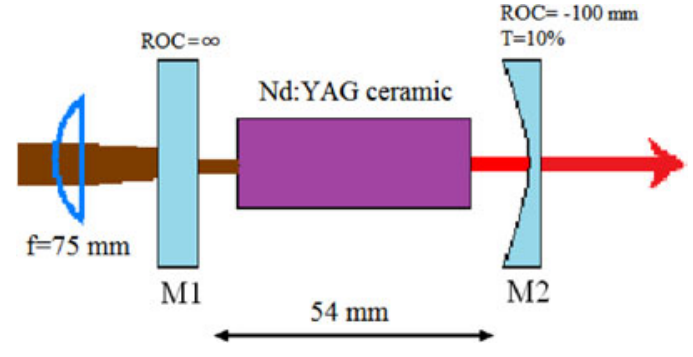

Figure 7. A schematic of the Nd:YAG ceramic laser setup.

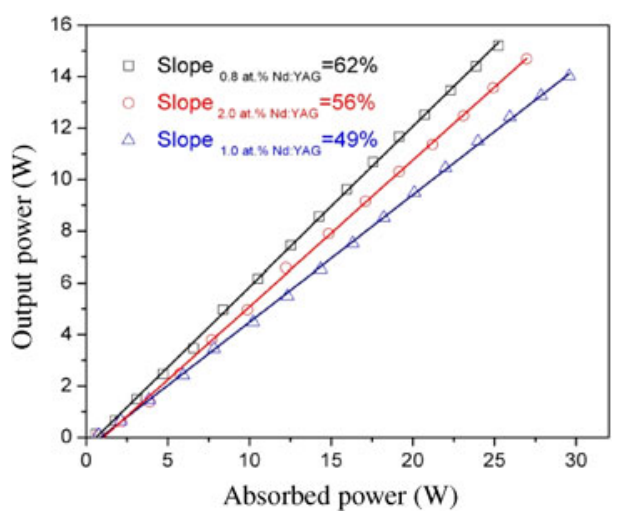

Figure 8. Laser performance of the Nd:YAG ceramic lasers.

to an absorption cross-section of $6.26 \times 10^{-20} \mathrm{~cm}^{2}$. From 900 to $1200 \mathrm{~nm}$, there are two main emission bands centered at 946 and $1064 \mathrm{~nm}$, which correspond to the ${ }^{4} \mathrm{~F}_{3 / 2} \rightarrow{ }^{4} \mathrm{I}_{9 / 2}$ and ${ }^{4} \mathrm{~F}_{3 / 2} \rightarrow{ }^{4} \mathrm{I}_{11 / 2}$ transitions of the $\mathrm{Nd}^{3+}$ ions, respectively. The strongest emission peak is at $1064 \mathrm{~nm}$, which is same as that of Nd:YAG single crystals. Figure 7 shows a schematic of our diode-pumped Nd:YAG ceramic laser setup. The Nd:YAG ceramics were cut into pieces of dimension $3 \times 3 \times 5 \mathrm{~mm}^{3}$. Nd:YAG ceramics with three $\mathrm{Nd}^{3+}$ doping concentrations (0.8 at.\%, 1.0 at.\%, and 2.0 at.\%) were used. The laser performance of the Nd:YAG ceramics is shown in Figure 8. The best performance was achieved with the 0.8 at. $\% \mathrm{Nd}: Y A G$ ceramic. A maximum output power of 

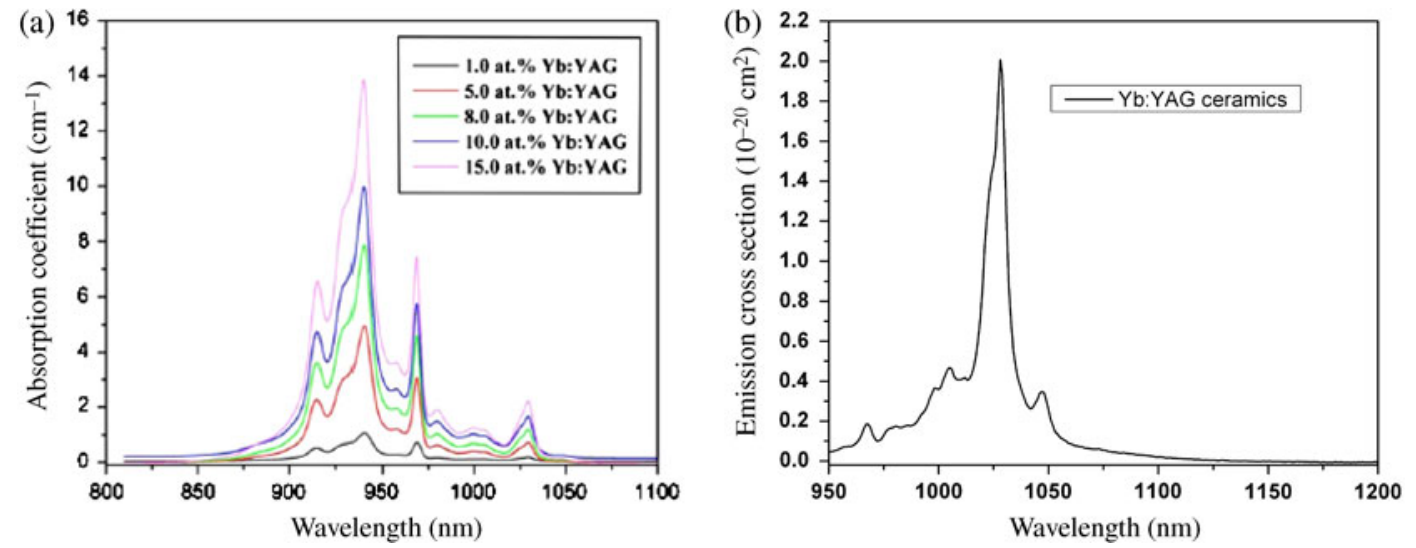

Figure 9. The absorption and emission spectra of the fabricated Yb:YAG ceramics.
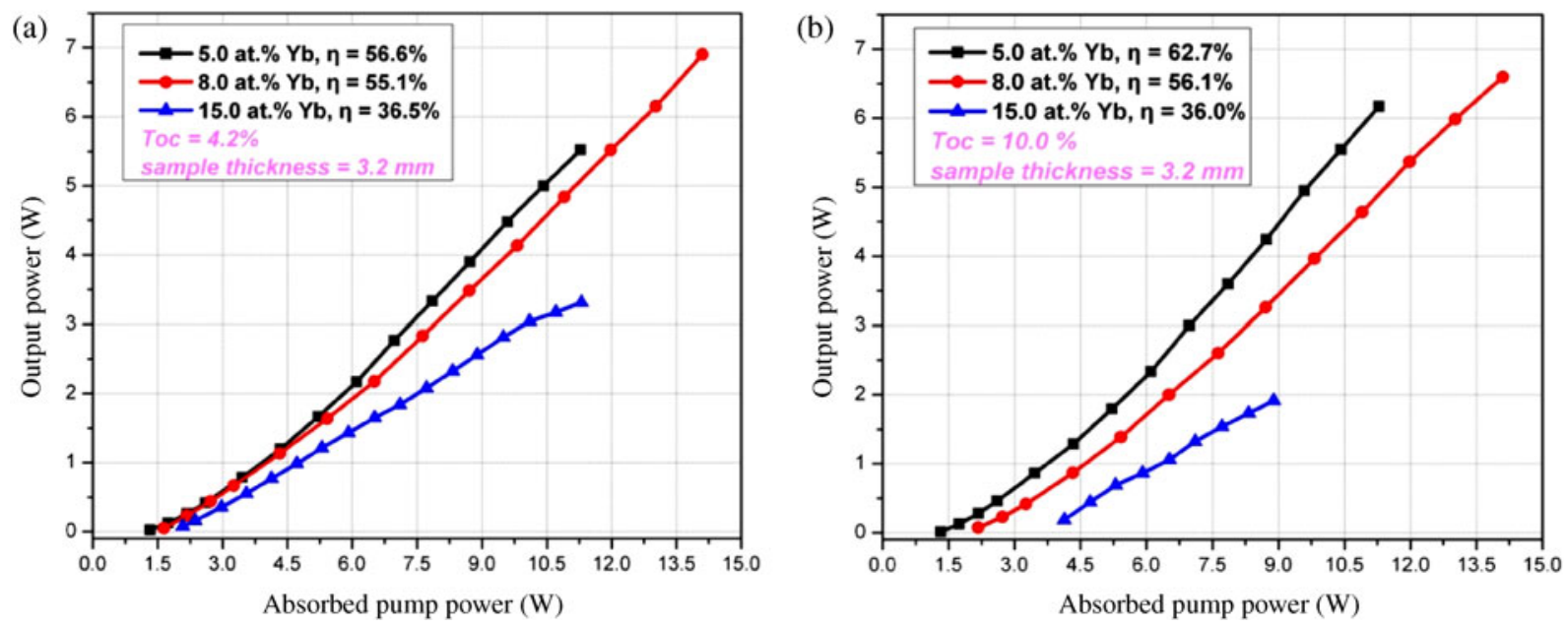

Figure 10. Laser performance of the Yb:YAG ceramic lasers.

$15.2 \mathrm{~W}$ at $1064 \mathrm{~nm}$ was obtained under an absorbed pump power of $25 \mathrm{~W}$. The slope efficiency is $62 \%$, which is very close to that of Nd:YAG single crystals.

The absorption spectra of the fabricated Yb:YAG ceramics under different $\mathrm{Yb}^{3+}$ doping concentrations are plotted in Figure 9(a). All the samples have a very strong absorption peak located at $940 \mathrm{~nm}$ and two moderate absorption peaks located at 915 and $969 \mathrm{~nm}$, respectively, corresponding to the ${ }^{2} \mathrm{~F}_{7 / 2} \rightarrow{ }^{2} \mathrm{~F}_{5 / 2}$ transition of $\mathrm{Yb}^{3+}$. The absorption coefficient at $940 \mathrm{~nm}$ increased from $1.06 \mathrm{~cm}^{-1}$ under the 1.0 at. $\% \mathrm{Yb}^{3+}$ doping concentration to $13.87 \mathrm{~cm}^{-1}$ under the 15.0 at.\% doping concentration. Absorption at $1030 \mathrm{~nm}$ is clearly observable, which is attributed to the quasi-threelevel nature of the $\mathrm{Yb}$ doped gain media. Figure 9(b) shows the emission cross-section of the Yb:YAG ceramics. The peak is at $1028 \mathrm{~nm}$, and it has a value of $2.01 \times 10^{-20} \mathrm{~cm}^{2}$. The laser performance of the Yb:YAG ceramics under two different output couplings $\left(T_{o c}\right)$ was experimentally studied. Yb:YAG ceramics with different doping concentrations (5.0 at.\%, 8.0 at.\%, and 15.0 at.\% ) were cut into pieces of dimension $3 \times 3 \times 3.2 \mathrm{~mm}^{3}$. The $\mathrm{CW}$ laser emission was at $1030 \mathrm{~nm}$. Under $T_{o c}=4.2 \%$, the laser threshold for the 5.0 at. $\%, 8.0$ at. $\%$, and 15.0 at. $\%$ Yb:YAG ceramics was $1.31 \mathrm{~W}, 1.64 \mathrm{~W}$, and $2.08 \mathrm{~W}$, respectively. Under $T_{o c}=10 \%$, it was $1.3 \mathrm{~W}, 2.16 \mathrm{~W}$, and $4.21 \mathrm{~W}$, respectively, as shown in Figure 10(a) and (b). Due to the reabsorption of the laser, the output power did not increase linearly with the absorbed pump power, but has the tendency that the larger the absorbed pump power, the higher the slope efficiency. With the experimentally available pump power, a maximum output power of $6.9 \mathrm{~W}$ was achieved on the 8.0 at.\% sample under $T_{o c}=4.2 \%$, while the highest slope efficiency of $62.7 \%$ was obtained with the 5.0 at.\% Yb:YAG ceramic sample under $T_{o c}=10 \%$. No saturation was observed on all the Yb:YAG ceramic lasers. We believe that, with further optimization of the doping concentration and experimental conditions, even higher output power and slope efficiency could be obtained.

As there is no concentration quenching for the $\mathrm{Yb}^{3+}$ doped laser materials, high doping is preferred to achieve high power and high efficiency of $\mathrm{Yb}^{3+}$ doped lasers. However, a problem associated with $\mathrm{Yb}^{3+}$ doped YAG is that, as the $\mathrm{Yb}^{3+}$ doping concentration is increased, the thermal conductivity of the doped YAG drops significantly. To solve the problem, the compound material $\mathrm{Lu}_{3} \mathrm{Al}_{5} \mathrm{O}_{12}$ 

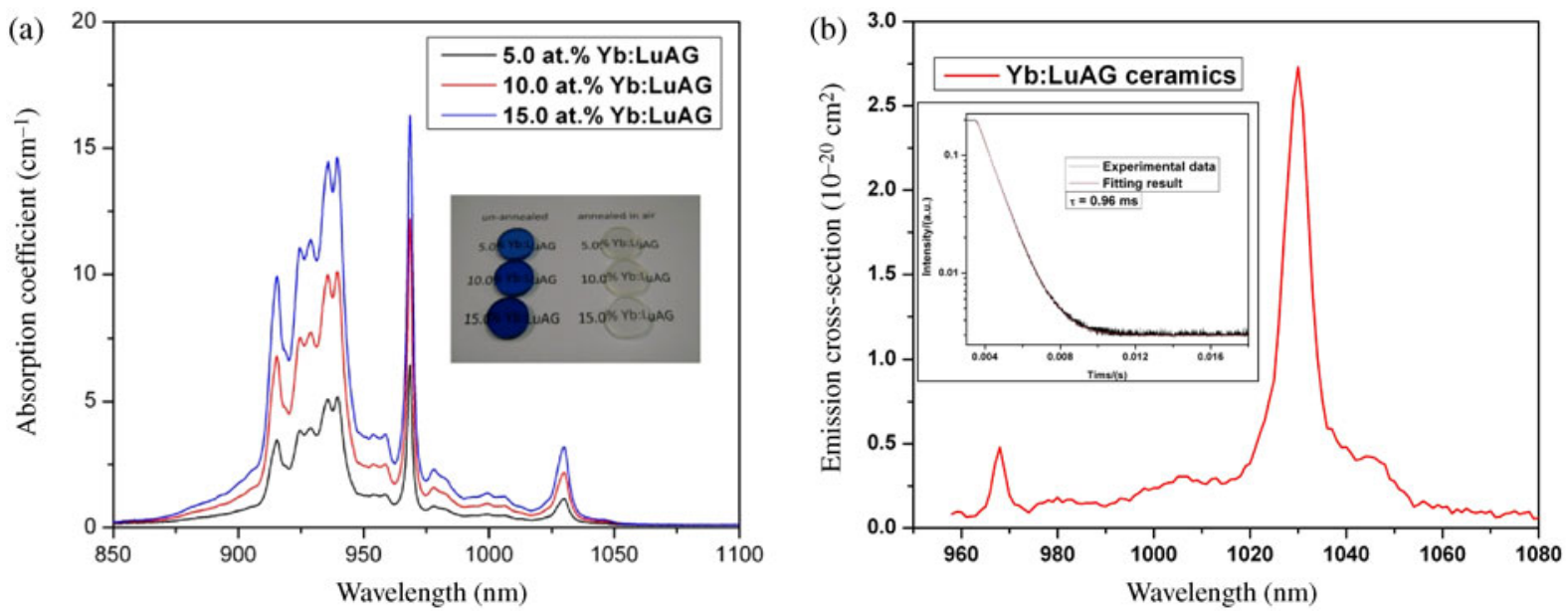

Figure 11. The absorption and emission spectra of the fabricated $\mathrm{Yb}$ :LuAG ceramics.

was proposed as an alternative to YAG. $\mathrm{Lu}_{3} \mathrm{Al}_{5} \mathrm{O}_{12}$ has high density $\left(6.72 \mathrm{~g} \mathrm{~cm}^{-3}\right)$, high thermal conductivity $(9.6 \mathrm{w} / \mathrm{m}$. $\mathrm{K})$, and a cubic structure. Although the thermal conductivity of LuAG is a little bit smaller than that of YAG, its conductivity decreases only slightly under high $\mathrm{Yb}$ doping, which is of benefit to high-power laser operation. We have also fabricated high optical quality $\mathrm{Yb}: \mathrm{LuAG}$ ceramics with different $\mathrm{Yb}^{3+}$ doping concentrations ${ }^{[29]}$ Figure 11(a) shows the absorption spectra of the as-fabricated $\mathrm{Yb}: \mathrm{LuAG}$ ceramic samples with different $\mathrm{Yb}^{3+}$ doping concentrations. A photograph of the $\mathrm{Yb}: \mathrm{LuAG}$ samples is also shown in the inset of the figure. The samples appear blue in color before annealing. After being annealed in air they become colorless and fully transparent. The absorption spectra of the Yb:LuAG ceramics are similar in profile to those of the Yb:YAG ceramics. However, the highest absorption peak is located at $968.4 \mathrm{~nm}$. For the samples with $5 \%, 10 \%$, and $15 \%$ at. $\% \mathrm{Yb}^{3+}$ concentrations, the peak absorption coefficients were measured as $6.4 \mathrm{~cm}^{-1}, 12.2 \mathrm{~cm}^{-1}$, and $16.3 \mathrm{~cm}^{-1}$, respectively. The highest absorption peak of $\mathrm{Yb}: \mathrm{LuAG}$ is very sharp, with a full width at half maximum (FWHM) of $2.5 \mathrm{~nm}$. The second highest absorption peak is at $940 \mathrm{~nm}$; it is a band consisting of double peaks, centered at $935.6 \mathrm{~nm}$ and $939.6 \mathrm{~nm}$, respectively. The two peaks have nearly the same absorption coefficient. The FWHM of the absorption band is $21.6 \mathrm{~nm}$, which favors direct diode pumping with InGaAs laser diodes. The absorption crosssection at the 939 and $968 \mathrm{~nm}$ absorption peaks is calculated to be $0.73 \times 10^{-20} \mathrm{~cm}^{2}$ and $0.91 \times 10^{-20} \mathrm{~cm}^{2}$, respectively. In addition, there is an absorption peak located at $1030 \mathrm{~nm}$, which is clearly observable on the spectrum. Figure 11(b) shows the emission cross-section and the fluorescent decay curve (inset) for a 5.0 at.\% Yb:LuAG ceramic. The emission peak of the Yb:LuAG ceramic is also located at the $1030 \mathrm{~nm}$ wavelength, whose emission cross-section is calculated to be $2.7 \times 10^{-20} \mathrm{~cm}^{2}$ using the Füchtbauer-Ladenburg $(\mathrm{F}-\mathrm{L})$ equation. The value is almost $30 \%$ higher than that of Yb:YAG $\left(\sim 2.0 \times 10^{-20} \mathrm{~cm}^{2}\right)$. The fluorescence decay of

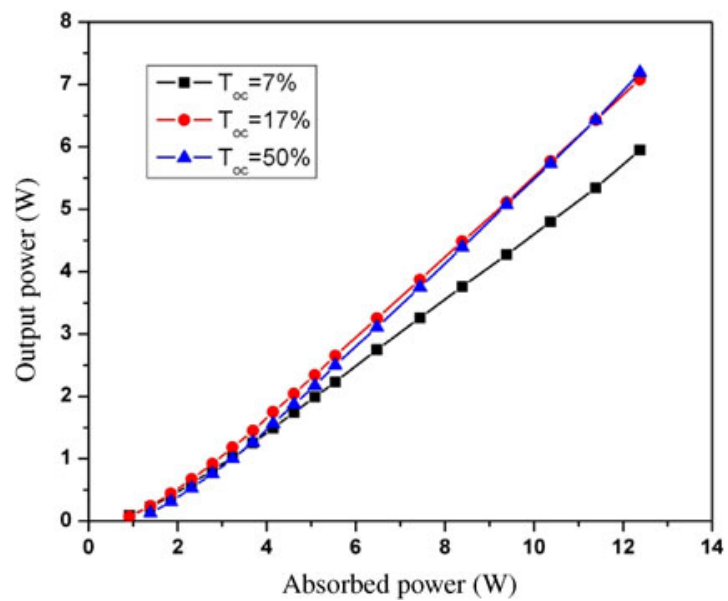

Figure 12. Laser performance of the Yb:LuAG ceramic lasers.

the $\mathrm{Yb}^{3+}$ ions in the LuAG ceramic sample was measured; the results are shown in the inset of Figure 11(b). A single exponential decay curve was observed. It gives a lifetime of $0.96 \mathrm{~ms}$. The $\mathrm{CW}$ laser performance of a 5.0 at.\% $\mathrm{Yb}: \mathrm{LuAG}$ ceramic was experimentally investigated under different output couplings. The Yb:LuAG samples were cut into pieces of dimension $3 \times 3 \times 3.2 \mathrm{~mm}^{3}$. The result is shown in Figure 12. Under Toc $=50 \%$, a maximum output power of $7.2 \mathrm{~W}$ was obtained at an absorbed pump power of $12.4 \mathrm{~W}$, corresponding to a slope efficiency of $65 \%$.

\section{2. $1.6 \mu \mathrm{m}$ laser oscillation}

The laser performance of the $\mathrm{Er}^{3+}$ doped YAG ceramics was also experimentally investigated. Figure 13(a) shows the absorption spectra of the Er:YAG ceramics under different doping concentrations ${ }^{[25]}$. The absorption coefficient of the Er:YAG ceramics increased with the increase of the $\mathrm{Er}^{3+}$ concentration. At $1532 \mathrm{~nm}$, the absorption coefficient increased from 0.64 to $5.06 \mathrm{~cm}^{-1}$ as the $\mathrm{Er}^{3+}$ concentration increased from 0.5 at. $\%$ to 5.0 at.\%. Figure 13(b) shows the 

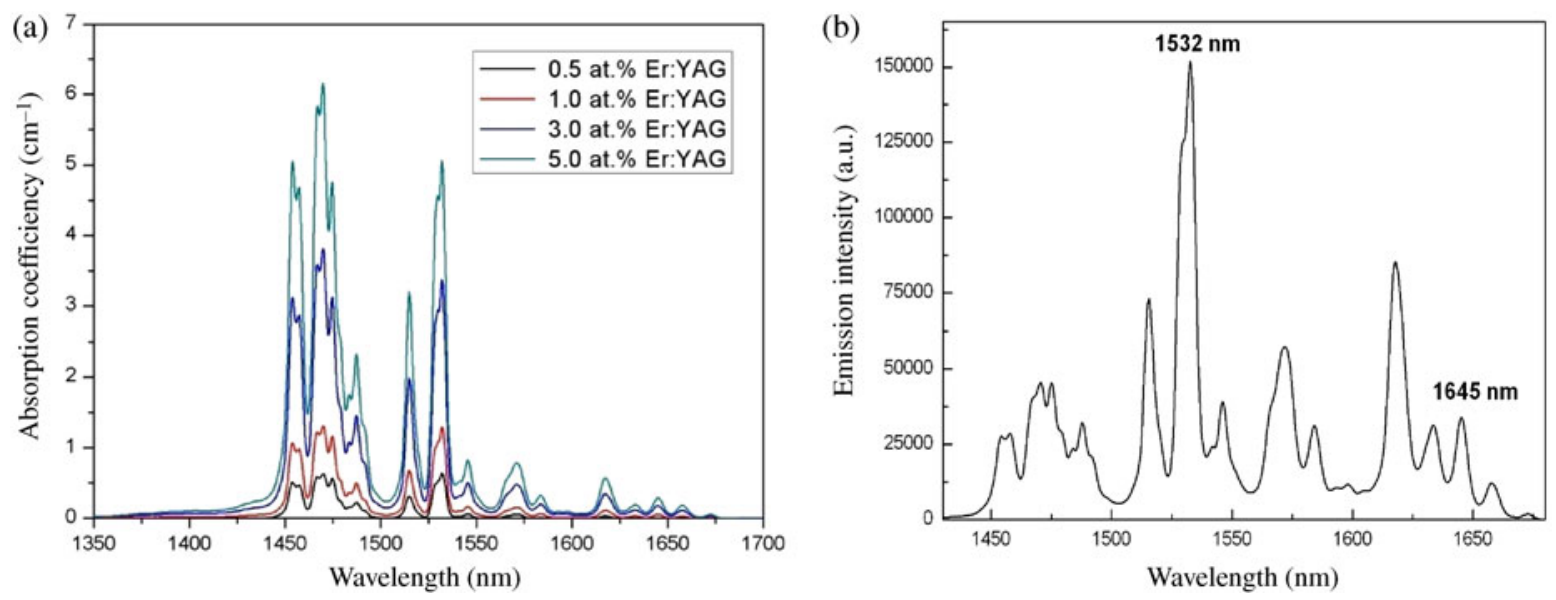

Figure 13. The absorption and emission spectra of the fabricated Er:YAG ceramics.

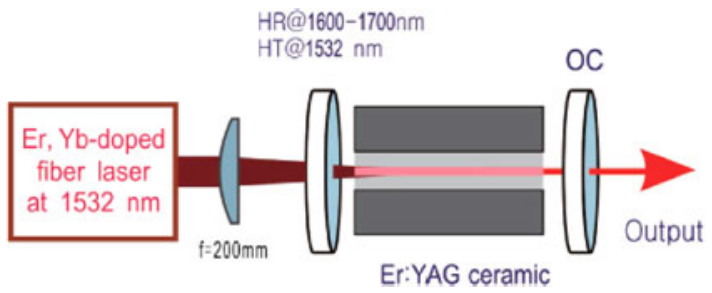

Figure 14. A schematic of the Er:YAG ceramic laser setup.

emission spectrum of the Er:YAG ceramic with a doping concentration of 0.5 at. $\%$ in the range $1400-1700 \mathrm{~nm}$. The emission is due to the ${ }^{4} \mathrm{I}_{13 / 2} \rightarrow{ }^{4} \mathrm{I}_{15 / 2}$ transition of the $\mathrm{Er}^{3+}$ ions. The highest emission peak is at $1532 \mathrm{~nm}$, with an emission cross-section of $0.99 \times 10^{-20} \mathrm{~cm}^{2}$. Figure 14 shows the schematic of the Er:YAG ceramic laser setup used in our experiment. The laser is in-band pumped by an $\mathrm{Er}-\mathrm{Yb}$ fiber laser at $1532 \mathrm{~nm}$. A 1.0 at.\% Er:YAG ceramic sample, cut into pieces of dimension $2 \times 4 \times 14 \mathrm{~mm}^{3}$, was used as the gain medium. An output coupler with $10 \%$ transmission at $1645 \mathrm{~nm}$ was used. The performance of the Er:YAG laser is shown in Figure 15. The laser has a pump threshold of $\sim 1.3 \mathrm{~W}$. It reached an output power of $13.8 \mathrm{~W}$ at $1645 \mathrm{~nm}$ under $27.3 \mathrm{~W}$ of incident pump power. A slope efficiency with respect to the incident pump power of $54.5 \%$ and an optical-optical conversion efficiency of $50.5 \%$ were achieved. No power saturation was observed on the maximum available pump power, suggesting that further power scaling of the laser is possible by simply increasing the input pumping power.

\section{3. $2.0 \mu \mathrm{m}$ laser oscillation}

Figure 16 shows the absorption and emission spectra of the Ho:YAG ceramics fabricated in-house ${ }^{[18]}$. As shown, the strongest absorption peak is at $1907 \mathrm{~nm}$, which is the same as that measured with the Ho:YAG single crystals. As the $\mathrm{Ho}^{3+}$ doping concentration increased from 0.8 to 2.0 at.\%, the absorption coefficient at $1907 \mathrm{~nm}$ increased linearly from 0.72 to $1.78 \mathrm{~cm}^{-1}$. Based on the measured
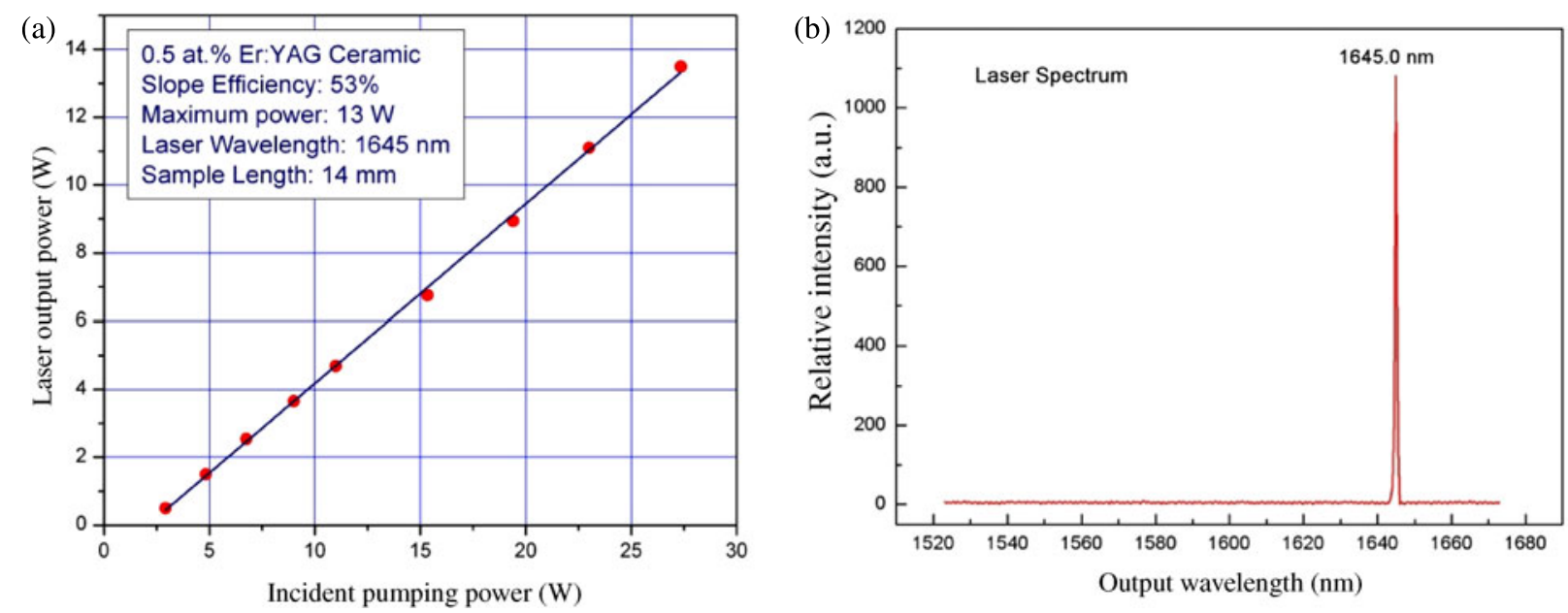

Figure 15. Laser performance of the Er:YAG ceramic laser. 

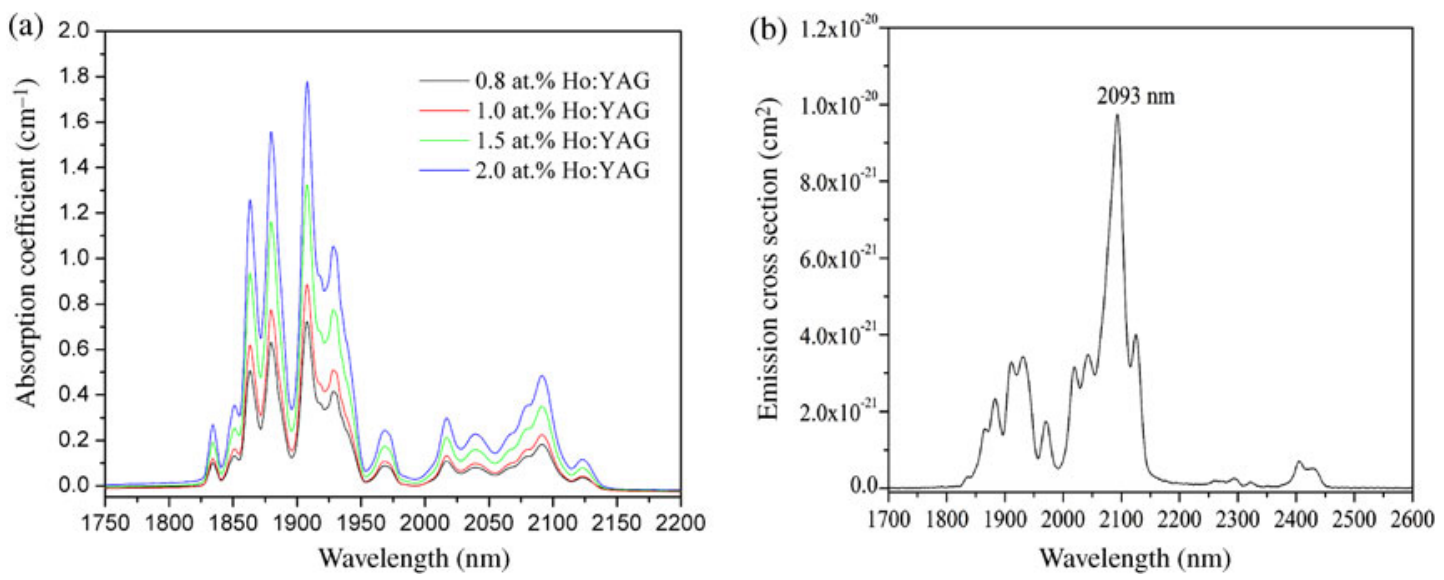

Figure 16. The absorption and emission spectra of the fabricated Ho:YAG ceramics.

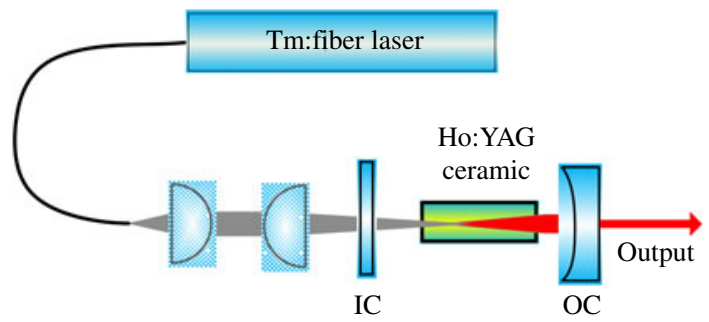

Figure 17. A schematic of the Ho:YAG ceramics laser setup.

absorption coefficients, the absorption cross-section of the Ho:YAG ceramic is calculated as $0.645 \times 10^{-20} \mathrm{~cm}^{2}$. The emission spectrum from 1700 to $2600 \mathrm{~nm}$ of a 1.0 at.\% Ho:YAG ceramic measured at room temperature is shown in Figure 16(b). The main emission band is centered at $2093 \mathrm{~nm}$, which corresponds to the ${ }^{5} \mathrm{I}_{7} \rightarrow{ }^{5} \mathrm{I}_{8}$ transition of $\mathrm{Ho}^{3+}$. The emission cross-section at $2093 \mathrm{~nm}$ is calculated to be $0.97 \times 10^{-20} \mathrm{~cm}^{2}$ by using the $\mathrm{F}-\mathrm{L}$ equation. Figure 17 shows a schematic of the Ho:YAG ceramic laser experimentally used. The Ho:YAG ceramic laser is in-band pumped by a home-made Tm:YAG fiber laser at $1907 \mathrm{~nm}$. The dimensions of the Ho:YAG ceramic used were $2 \mathrm{~mm} \times$ $3 \mathrm{~mm} \times 14 \mathrm{~mm}$. A 1.5 at.\% Ho:YAG ceramic was used as the laser gain medium. With $35 \mathrm{~W}$ incident pump power, a maximum output power of $20.6 \mathrm{~W}$ at $2097 \mathrm{~nm}$ was obtained, corresponding to a slope efficiency of $60.9 \%$ and an optical-to-optical conversion efficiency of $58.8 \%$, as shown in Figure 18.

Besides Ho:YAG ceramics, Tm:YAG ceramics also emit in the $2 \mu \mathrm{m}$ wavelength regime. Figure 19 shows the transmittance and emission spectrum of the in-house fabricated Tm:YAG ceramics. There are six strong absorption bands centered at 356, 458, 684, 781, 1202, and $1626 \mathrm{~nm}$, which correspond to the transitions of $\mathrm{Tm}^{3+}$ from the ground state ${ }^{3} \mathrm{H}_{6}$ to its excited state, ${ }^{1} \mathrm{D}_{2},{ }^{1} \mathrm{G}_{4},{ }^{3} \mathrm{~F}_{3},{ }^{3} \mathrm{H}_{4},{ }^{3} \mathrm{H}_{5}$, and ${ }^{3} \mathrm{~F}_{4}$, respectively. The strong emission peaks are at wavelengths of $1626 \mathrm{~nm}, 1702 \mathrm{~nm}, 1746 \mathrm{~nm}, 1785 \mathrm{~nm}, 1882 \mathrm{~nm}$, $1960 \mathrm{~nm}$, and $2016 \mathrm{~nm}$, respectively. Tm:YAG is a quasithree-level system. Hence, laser emission with wavelength
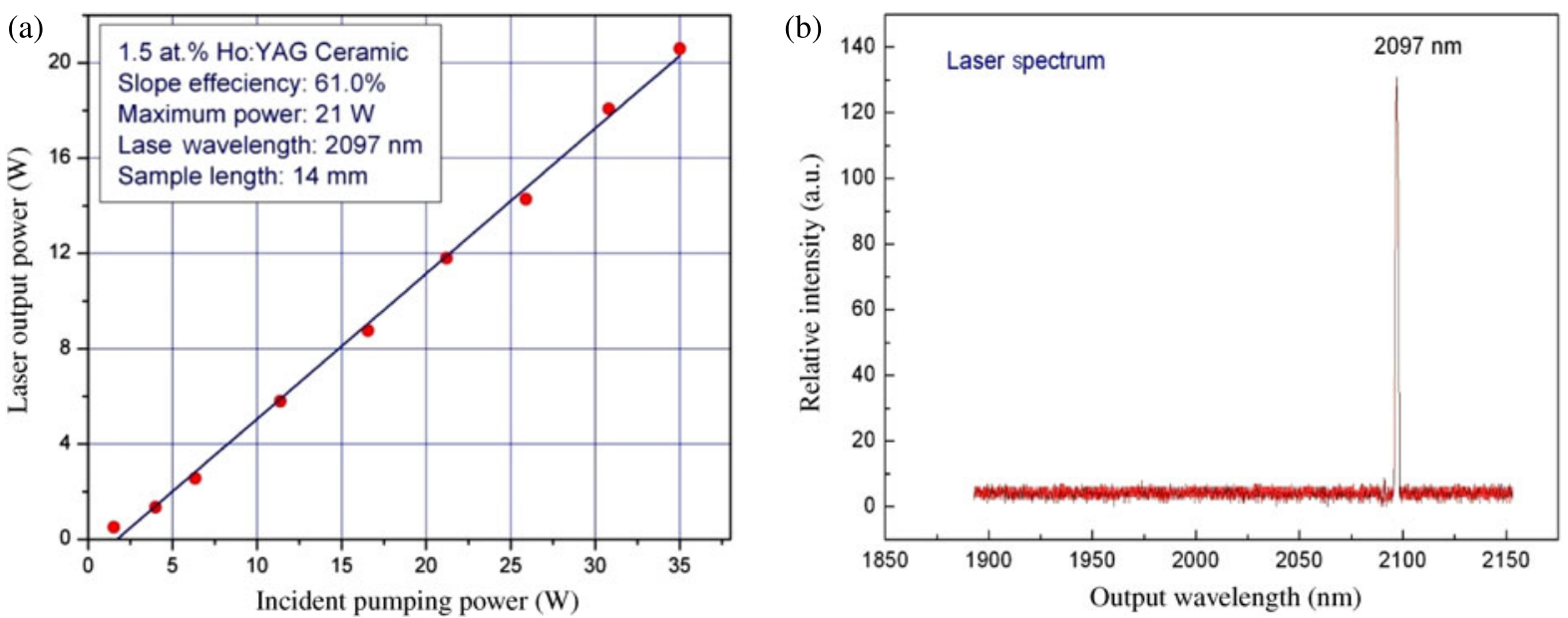

Figure 18. Laser performance of the Ho:YAG ceramic laser. 

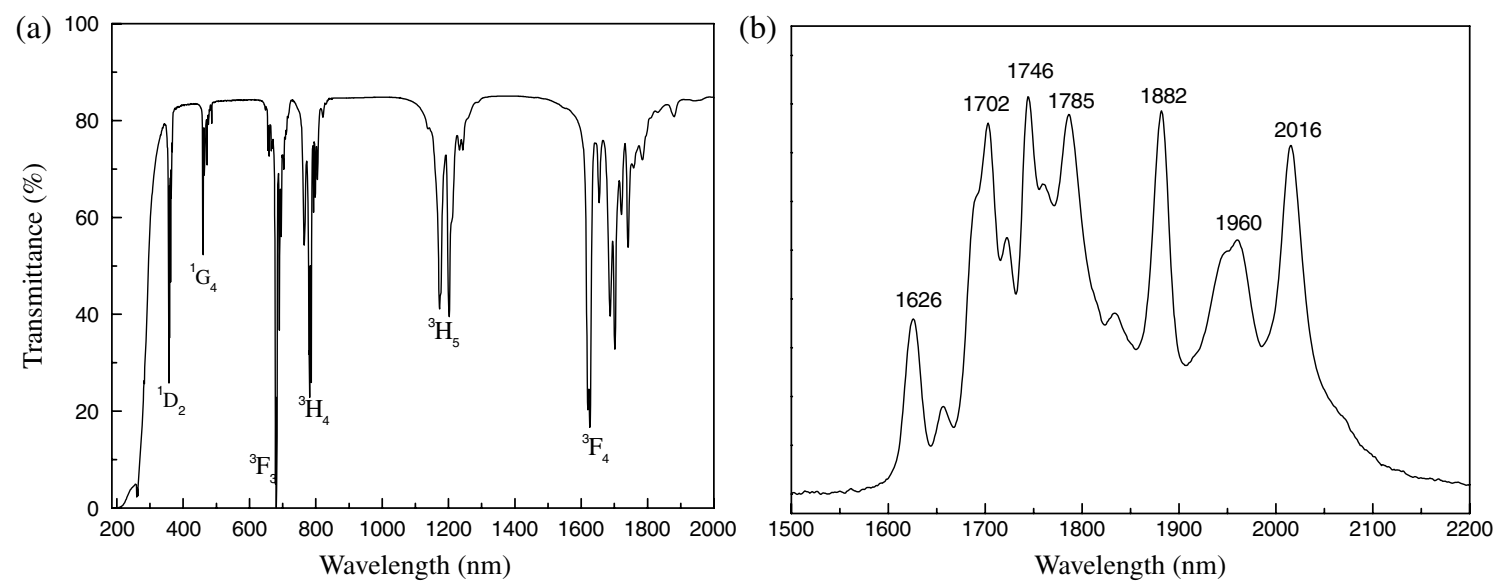

Figure 19. The transmittance and emission spectra of a fabricated Tm:YAG ceramic.

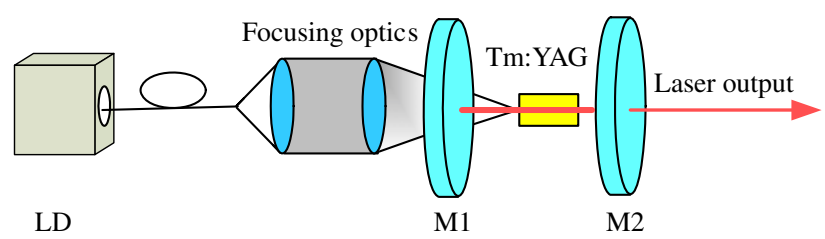

Figure 20. A schematic of the diode-pumped Tm:YAG ceramic laser setup.

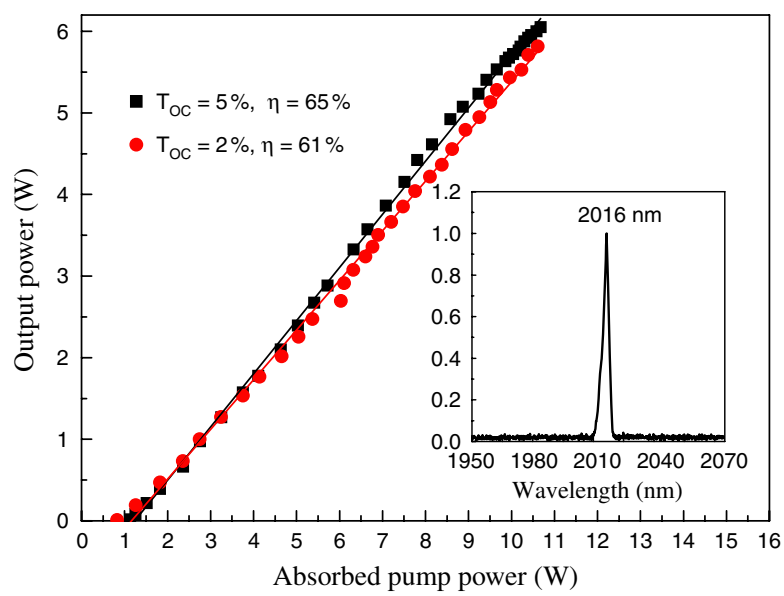

Figure 21. Laser performance of the Tm:YAG ceramic lasers.

below $1.9 \mu \mathrm{m}$ is difficult to achieve, due to the reabsorption by the $\mathrm{Tm}^{3+}$ ions.

Tm:YAG lasers can be directly pumped by commercially available high-power GaAlAs diodes at $\sim 800 \mathrm{~nm}$ (the ${ }^{3} \mathrm{H}_{6} \rightarrow{ }^{3} \mathrm{H}_{4}$ transition). Benefitting from a fortuitous 'two-for-one' cross-relaxation process, pumping quantum efficiencies approaching 2 could be achieved with pumping under high $\mathrm{Tm}^{3+}$ doping concentrations. Figure 20 shows the schematic of the diode-pumped Tm:YAG ceramic laser used to study the lasing performance of the fabricated Tm:YAG ceramics. The diode laser is operating at $786 \mathrm{~nm}$. Tm:YAG ceramics with different doping concentrations were cut into pieces of dimension $3 \times 3 \times 3.2 \mathrm{~mm}^{3}$. The laser emitted at $2016 \mathrm{~nm}$. With an output coupler of 5\% coupling, a maximum output power of $6.05 \mathrm{~W}$ was obtained under an absorbed pump power of $10.68 \mathrm{~W}$, giving a slope efficiency of $65 \%$.

In Table 1, we have summarized the laser performance of our fabricated RE:YAG ceramics.

\section{Conclusions}

High optical quality polycrystalline YAG transparent ceramics doped with various rare-earth ions were successfully fabricated by the reactive sintering method. The laser performance of the fabricated RE:YAG ceramics were studied experimentally. The main results achieved in our works can be summarized as follows.

(1) High-quality RE:YAG transparent ceramics were fabricated using the reactive sintering method under vacuum conditions. Irrespective of the different doped rare-earth ions and their doping levels, defect-free microstructures were observed for all the samples produced.

(2) Highly efficient CW laser oscillations in the $1.0 \mu \mathrm{m}$ regime were obtained in the Nd:YAG and Yb:YAG ceramics.

Table 1. Summary of the results of the ceramic laser experiments

\begin{tabular}{lllllll}
\hline & Nd:YAG & Yb:YAG & Yb:LuAG & Er:YAG & Tm:YAG & Ho:YAG \\
\hline Pumping wavelength & $808 \mathrm{~nm}$ & $940 \mathrm{~nm}$ & $980 \mathrm{~nm}$ & $1532 \mathrm{~nm}$ & $786 \mathrm{~nm}$ & $1907 \mathrm{~nm}$ \\
Output wavelength & $1064 \mathrm{~nm}$ & $1030 \mathrm{~nm}$ & $1030 \mathrm{~nm}$ & $1645 \mathrm{~nm}$ & $2016 \mathrm{~nm}$ & $2097 \mathrm{~nm}$ \\
Maximum output power & $15 \mathrm{~W}$ & $6.9 \mathrm{~W}$ & $7.2 \mathrm{~W}$ & $13.8 \mathrm{~W}$ & $6.05 \mathrm{~W}$ & $21 \mathrm{~W}$ \\
Slope efficiency & $62.0 \%$ & $62.7 \%$ & $65.0 \%$ & $54.5 \%$ & $65.0 \%$ & $61.0 \%$ \\
\hline
\end{tabular}


The highest slope efficiency achieved on the Nd:YAG ceramics is $62 \%$, which is very close to that of Nd:YAG single crystal.

(3) Er:YAG ceramic is suitable for achieving $\sim 1.6 \mu \mathrm{m}$ laser emission. When end pumped by an Er,Yb-fiber laser at $1532 \mathrm{~nm}$, a maximum output power of $13.8 \mathrm{~W}$ at $1645 \mathrm{~nm}$ was obtained with a slope efficiency of $54.5 \%$.

(4) By using a 1.5 at.\% Ho:YAG ceramic as the laser medium, 20.6 W laser output at $2097 \mathrm{~nm}$ was achieved under $35 \mathrm{~W}$ of incident pump power, and a slope efficiency of $61 \%$ has been achieved.

(5) High optical quality Tm:YAG laser ceramics have been fabricated. Pumped by a laser diode at $768 \mathrm{~nm}$, highefficiency $\mathrm{CW}$ laser operation has been demonstrated.

\section{Acknowledgements}

This project is supported by the Priority Academic Program Development of Jiangsu Higher Education Institutions (PAPD) and the National Research Foundation of Singapore under contract NRF-G-CRP-2007-01.

\section{References}

1. G. L. Messing, and A. J. Stevenson, Science 322, 383 (2008).

2. A. Ikesue, and Y. L. Aung, Nature Photonics 2, 721 (2008).

3. R. L. Coble, J. Amer. Ceram. Soc. 45, 123 (1962).

4. A. Ikesue, T. Kinoshita, K. Kamata, and K. Yoshida, J. Am. Ceram. Soc. 78, 1033 (1995).

5. A. Ikesue, I. Furusato, and K. Kamata, J. Am. Ceram. Soc. 78, 225 (1995).

6. A. Ikesue, K. Kamata, and K. Yoshida, J. Am. Ceram. Soc. 78, 2545 (1995)

7. J. R. Lu, M. Prabhu, J. Song, et al., Appl. Phys. B 71, 469 (2000).

8. J. R. Lu, J. Song, M. Prabhu, et al., Jpn. J. Appl. Phys 39, L1048 (2000).

9. J. Lu, K. Ueda, H. Yagi, T. Yanagitani, Y. Akiyama, and A. A. Kaminskii, J. Alloy. Compd. 341, 220 (2002).
10. J. Lu, T. Murai, K. Takaichi, et al., Appl. Phys. Lett. 78, 3586 (2001).

11. J. Lu, H. Yagi, K. Takaichi, et al., Appl. Phys. B 79, 25 (2004).

12. L. Maziex, and D. J. Green, Int. J. Appl. Ceram. Technol. 3, 166 (2006).

13. H. Yagi, K. Takaichi, K. Ueda, Y. Yamasaki, T. Yanagitani, and A. A. Kaminskii, Laser Phys. 15, 1338 (2005).

14. R. M. Yamanoto, Proc. Adv. Solid State Photon., Nara, Japan, WC5 (2008).

15. B Bishop, "Northrop Grumman scales new heights in electric laser power, achieves $100 \mathrm{~kW}$ from a solid-state laser", http://www.irconnect.com/noc/press/pages/news_releases.html (March 18, 2009).

16. A. Mandl, and D. E. Klimek, in Conference on Lasers and Electro-Optics, OSA Technical Digest (CD) (Optical Society of America 2010), paper JThH2.

17. R. Guoguang, and H. Yunian, Laser \& Infrared 36, 617 (2006).

18. J. Dong, G. Z. Xu, J. Ma, et al., Opt. Mater. 34, 959 (2012).

19. W. Kim, C. Baker, G. Villalobos, et al., J. Am. Ceram. Soc. 94, 3001 (2011).

20. E. R. Kupp, G. L. Messing, J. M. Anderson, and V. Gopalan, J. Mater. Res. 25, 476 (2010).

21. H. Yagi, T. Yanagitani, K. Takaichi, K. Ueda, and A. A. Kaminskii, Opt. Mater. 29, 1258 (2007).

22. H. Gong, D. Y. Tang, H. Huang, and J. Ma, J. Am. Ceram. Soc. 92, 812 (2009).

23. H. Yang, X. P. Qin, J. Zhang, et al., Opt. Mater. 34, 940 (2012).

24. D. W. Luo, J. Zhang, C. W. Xu, X. P. Qin, D. Y. Tang, and J. Ma, Opt. Mater. 34, 936 (2012).

25. X. P. Qin, H. Yang, G. H. Zhou, et al., Opt. Mater. 34, 973 (2011).

26. Y. Wang, D. Y. Shen, H. Chen, et al., Opt. Lett. 36, 4485 (2011).

27. H. Yang, J. Zhang, X. P. Qin, et al., J. Am. Ceram. Soc. 95, 52 (2012).

28. W. L. Gao, J. Ma, G. Q. Xie, et al., Opt. Lett. 37, 1076 (2012).

29. C. W. Xu, D. W. Luo, J. Zhang, et al., Laser Phys. Lett. 9, 30 (2012). 